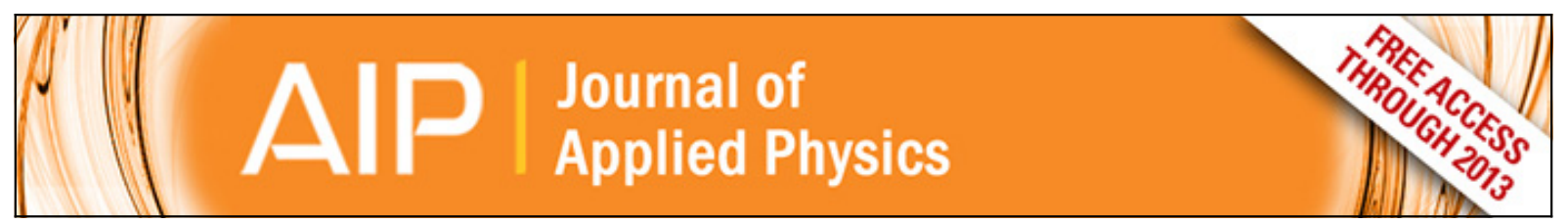

\title{
Cyclic electric field stress on bipolar resistive switching devices
}

A. Schulman and C. Acha

Citation: Journal of Applied Physics 114, 243706 (2013); doi: 10.1063/1.4859475

View online: http://dx.doi.org/10.1063/1.4859475

View Table of Contents: http://scitation.aip.org/content/aip/journal/jap/114/24?ver=pdfcov

Published by the AIP Publishing

AlPP Re-register for Table of Content Alerts

Create a profile.

Sign up today! 


\title{
Cyclic electric field stress on bipolar resistive switching devices
}

\author{
A. Schulman ${ }^{\text {a) }}$ and C. Acha ${ }^{\text {b) }}$ \\ Laboratorio de Bajas Temperaturas, Departamento de Física, FCEyN - Universidad de Buenos Aires \\ and IFIBA - CONICET, Pabellón I, Ciudad Universitaria, C1428EHA Buenos Aires, Argentina
}

(Received 3 October 2013; accepted 12 December 2013; published online 27 December 2013)

\begin{abstract}
We have studied the effects of accumulating cyclic electrical pulses of increasing amplitude on the non-volatile resistance state of interfaces made by sputtering a metal (Au, $\mathrm{Pt}$ ) on top of the surface of a cuprate superconductor $\mathrm{YBa}_{2} \mathrm{Cu}_{3} \mathrm{O}_{7-\delta}$. We have analyzed the influence of the number of applied pulses $N$ on the relative amplitude of the remnant resistance change between the high $\left(R_{H}\right)$ and the low $\left(R_{L}\right)$ state $\left[\left(\alpha=\left(R_{H}-R_{L}\right) / R_{L}\right]\right.$ at different temperatures $(T)$. We show that the critical voltage $\left(V_{c}\right)$ needed to produce a resistive switching (RS, i.e., $\left.\alpha>0\right)$ decreases with increasing $N$ or $T$. We also find a power law relation between the voltage of the pulses and the number of pulses $N_{\alpha_{0}}$ required to produce a $\mathrm{RS}$ of $\alpha=\alpha_{0}$. This relation remains very similar to the Basquin equation used to describe the stress-fatigue lifetime curves in mechanical tests. This points out to the similarity between the physics of the RS, associated with the diffusion of oxygen vacancies induced by electrical pulses, and the propagation of defects in materials subjected to repeated mechanical stress. (C) 2013 AIP Publishing LLC. [http://dx.doi.org/10.1063/1.4859475]
\end{abstract}

\section{INTRODUCTION}

The search of new non-volatile memories is reinforced nowadays by the necessity of producing more dense, less dissipative, and low cost devices. ${ }^{1}$ Memories based on the resistive switching (RS) mechanism on metal-oxide interface are marked as one of the most promising candidates for the next generation of memory applications. ${ }^{2,3}$ A typical RS device consists of an interface between a metal and an oxide, which can be either in a capacitor-like form or in a planar structure. Depending on the oxide, the mechanism beneath the RS can give rise to a polarity-independent filamentary effect (typically observed for binary oxides) ${ }^{4}$ or to a polarity-sensitive one (observed for complex-oxides) associated with interfacial properties, ${ }^{5,6}$ although exceptions can be observed in both categories. ${ }^{7,8}$

While significant advances have been made in improving device performances, understanding their underlying physics still represents a great challenge. In addition to scalability, fast response, repeatability, retentivity, and low power consumption, endurance is one of the properties that these memories must fulfill. ${ }^{9}$ The cyclic electric field stress that implies the repeated switching of the device may produce an accumulation of defects that would affect its electrical properties. As during the normal operation of a device out of the range high and low resistance states can be produced, error correction techniques must be taken into account. ${ }^{10}$ These techniques can be based on feedback protocols in order to achieve, for example, a RS with resistance values in the desired range.

This is the particular point that we address in this paper, by analyzing the evolution of the remanent resistance of a bipolar device composed by metal-perovskite [(Au,Pt)-YBCO]

\footnotetext{
${ }^{a}$ University of Buenos Aires and CONICET of Argentina scholarships.

${ }^{b)}$ Author to whom correspondence should be addressed. Electronic mail: acha@df.uba.ar
}

junctions upon the application of a cyclic accumulation of pulses. In this type of devices, by considering that the resistance of the interface is proportional to the density of vacancies, the RS mechanism was associated with the electromigration of oxygen vacancies. ${ }^{11}$ Our results indicate that the mechanism that determines the evolution of the remnant resistance upon the application of a cyclic accumulation of pulses presents close similarities to the one governing the propagation of fractures during a mechanical-fatigue test on a material. ${ }^{12}$

\section{EXPERIMENTAL}

To study the dependence of the bipolar RS with cyclic electric field stress, we sputtered four metallic electrodes on one of the faces of a good quality YBCO textured ceramic sample (see the inset of Fig. 1). The width of the sputtered electrodes was in the order of $1 \mathrm{~mm}$ with a mean separation between them of $0.4 \mathrm{~mm}$ to $0.8 \mathrm{~mm}$. They cover the entire width of one of the faces of the YBCO slab $\left(8 \times 4 \times 0.5 \mathrm{~mm}^{3}\right)$. Silver paint was used to fix copper leads carefully without contacting directly the surface of the sample. Details about the synthesis and the RS characteristics of the metal-YBCO interfaces can be found elsewhere. ${ }^{11,13-18}$ We choose as metals Au and Pt for the pair of pulsed electrodes, labeled 1 and 2, respectively. As we have shown previously, the Pt-YBCO interfaces have a lower resistance value than the Au-YBCO ones $(R(P t) \lesssim R(A u) / 3)$, and a small RS amplitude. In this way, only the Au-YBCO (1) electrode will be active, simplifying the effects produced upon voltage pulsing treatments. After applying a burst of $N\left(10^{4} \leq N\right.$ $\left.\leq 5 \times 10^{5}\right)$ unipolar square voltage pulses $(100 \mu$ s width at $1 \mathrm{kHz}$ rate), the remnant resistance of each pulsed contact was measured using a small current through contacts 1-2 and a convenient set of additional Au contacts. To estimate the resistance $R(A u)$ and $R(P t)$, of the active Au-YBCO and of the Pt-YBCO interfaces, respectively, the voltage difference between electrodes 1-3 and 4-2 was measured. 


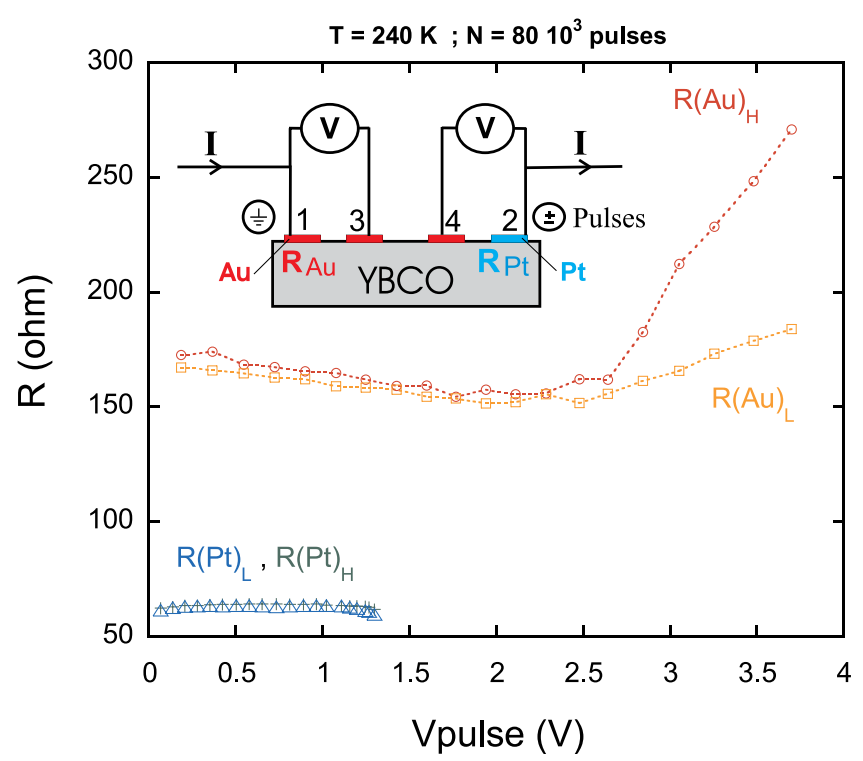

FIG. 1. Dependence with the amplitude of $N=80 \times 10^{3}$ square pulses $\left(V_{\text {pulse }}\right)$ of the remanent resistance of each contact (low state and high state for the $\mathrm{Au}$ and $\mathrm{Pt}$ interfaces, respectively). The $V_{\text {pulse }}$ magnitude is different for each contact as it corresponds to their effective voltage drop. $100 \mu \mathrm{s}$ pulses were applied at $1 \mathrm{kHz}$ rate at a constant temperature $(240 \mathrm{~K})$. The high and the low states of these contacts obtained after applying $-V_{\text {pulse }}^{\max }$ are also shown for comparison. Lines are guides to the eye.

Corrections to $R(A u)$ and $R(P t)$ by considering the resistance of the bulk YBCO are negligible taking into account that its value is only $\simeq 0.1 \Omega \ll 50 \Omega<R(A u), R(P t)$ in this temperature range. We want to note that the polarity of the pulses was defined arbitrarily with the ground terminal connected to the Au-YBCO contact. Temperature was measured with a Pt thermometer in the $200 \mathrm{~K}$ to $340 \mathrm{~K}$ range and stabilized better than at $0.5 \%$ after each pulsing treatment.

To perform a cyclic electric field stress experiment at a fixed temperature $T_{0}$, temperature is initially stabilized. As no electroforming step is needed, we initially set the active $\mathrm{Au}-\mathrm{YBCO}$ electrode to its low resistance state $\left(R(A u)_{L}\right)$ with a burst of pulses of $-5 \mathrm{~V}$ amplitude while, in a complementary manner, the Pt-YBCO electrode is in its high resistance state $\left(R(P t)_{H}\right)$. Note here that as the Au-YBCO electrode is the ground terminal a negative pulse indicates that its potential is higher than that of the Pt-YBCO electrode. Consequently, the density of oxygen vacancies near the $\mathrm{Au}-\mathrm{YBCO}$ interface should decrease (as they are positive charged defects), reducing the interfacial resistance, as we observe, in accordance to the voltage enhanced oxygen vacancy model that describes RS for bipolar devices. ${ }^{11}$

As mentioned previously, no relevant changes are expected in this electrode resistance $\left(R(P t)_{L} \simeq R(P t)_{H}\right)$. Then, we apply a "reset" burst of $N$ unipolar pulses with a $V_{\text {pulse }}$ amplitude during a time $t_{0}$ (from $10 \mathrm{~s}$ to $500 \mathrm{~s}$, depending on the $N$ value). Although the temperature is constantly stabilized to $T_{0}$, in order to avoid overheating effects on the resistance measurements, a time $t_{0}$ is waited before measuring the remnant resistance of each pulsed contact $\left(R(A u)_{H}\right.$ and $\left.R(P t)_{L}\right)$. After that, a "set" burst of maximum opposite polarity $\left(-V_{\text {pulse }}^{\max }=-5 \mathrm{~V}\right)$ is applied to subject the material to a cyclic stress. In this way, the resistance change is partially recovered and both remnant resistances are measured again $\left(R(A u)_{L}\right.$ and $\left.R(P t)_{H}\right)$. The process is then completely repeated for a new $V_{\text {pulse }}$ value, increased with a fixed step, until it reaches our experimental maximum $\left(V_{p u l s e}^{\max }=5 \mathrm{~V}\right)$.

\section{RESULTS AND DISCUSSION}

A typical result of a cyclic electric field stress experiment at a fixed number of pulses of the burst $(N=80 \mathrm{k}$ pulses) and temperature $(240 \mathrm{~K})$ is shown in Fig. 1, where we have plotted the remnant resistances of each pulsed electrode $(\mathrm{Au}$ and $\mathrm{Pt})$ in both high and low states as a function of the "reset" amplitude of the pulse $\left(V_{\text {pulse }}\right)$. $V_{\text {pulse }}$ corresponds to the voltage drop measured at each particular electrode. We can observe that, as expected, $R(P t)_{H} \simeq R(P t)_{L}$ for the smaller voltage drop explored, while $R(A u)_{H}>R(A u)_{L}$ for $V_{\text {pulse }}$ higher than a critical voltage $\left(V_{c}\right)$, indicating the existence of RS for this electrode. Hereafter, we will show results only for this $\mathrm{Au}-\mathrm{YBCO}$ active electrode.

The relative amplitude of the remnant resistance change between the high $\left(R_{H}\right)$ and the low $\left(R_{L}\right)$ state is defined as $\alpha=\left(R_{H}-R_{L}\right) / R_{L}=\Delta R / R_{L}$. It can be noted that while $\alpha \simeq 0$ for $V_{\text {pulse }} \leq V_{c}$ both $R(A u)_{H}$ and $R(A u)_{L}$ decrease with increasing $V_{\text {pulse }}$. This is a consequence of the protocol used for this particular cyclic treatment that forces $R(A u)_{L}$ to a lower value than the initial one, as for each reset burst of amplitude $V_{\text {pulse }}$ a set burst of amplitude $-V_{\text {pulse }}^{\max }$ is applied. This situation is reversed for $V_{\text {pulse }} \geq V_{c}$, where the increase in $R(A u)_{H}$ obtained is not completely recovered when the set protocol is applied.

The dependence of $\alpha$ with the amplitude $V_{\text {pulse }}$ of the burst of $N$ pulses at different temperatures can be depicted in Fig. 2. A noisy $\alpha<0.1$ is obtained until $V_{\text {pulse }} \geq V_{c}$, where $\alpha$ increases with $\left(V_{\text {pulse }}-V_{c}\right)$, following a power law-like behavior. As temperature is increased, $V_{c}$ decreases linearly and $\alpha$ reaches higher values. A similar behavior occurs when performing the experiment at a fixed temperature and increasing the number $N$ of pulses conforming the burst, as it is shown in Fig. 3. In this case, $\alpha$ and $V_{c}$ varies logarithmically with $N$ (not shown here). The former was also observed for Ag-manganite interfaces when $N$ pulses of the same polarity were accumulated. ${ }^{19}$

In a mechanical fatigue test, the material is subjected to a cyclic stress. Fatigue occurs when, for a stress $(S)$ above a certain threshold, a defect zone progresses along the material until a fracture occurs for $N_{\alpha}$ cycles, which corresponds to the number of cycles to failure. $S-N_{\alpha}$ curves (or Wöhler's curves ${ }^{20}$ ) can then be plotted in order to represent the lifetime of a material subjected to these conditions.

Here, for bipolar devices, it is known that the RS is associated with the electro-migration of vacancies in and out of the active metal-oxide interface. ${ }^{11}$ We can expect that a severe pulsing treatment may generate extended vacancy defects, but, as far as we know, in the electric field range explored, no macroscopic cracks were ever observed. ${ }^{21}$ So, it is necessary to define what can we call as an electric failure of the device. As possible scenarios, it can be expected that an arbitrary value of $\alpha$ can be obtained if the density of vacancies near the active interface (ai) can be reversibly 
a)

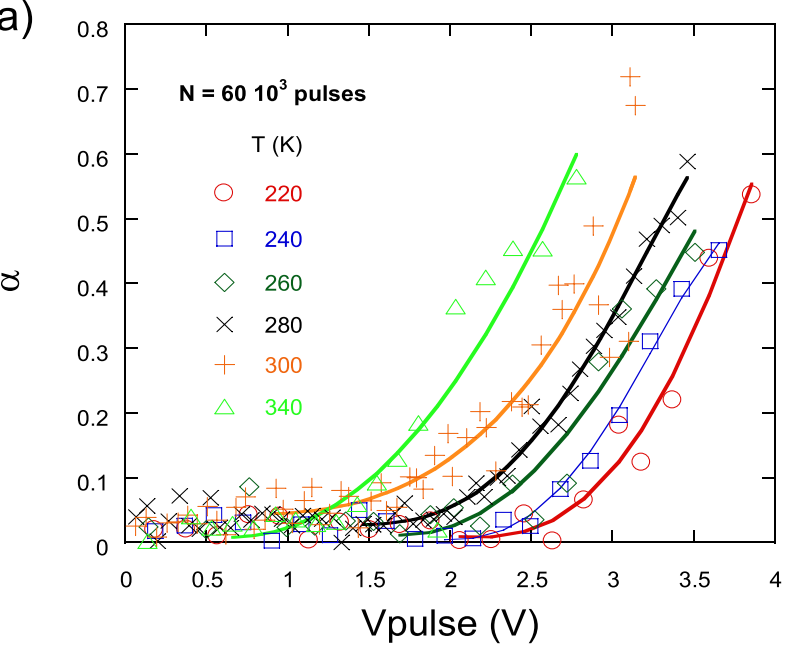

b)

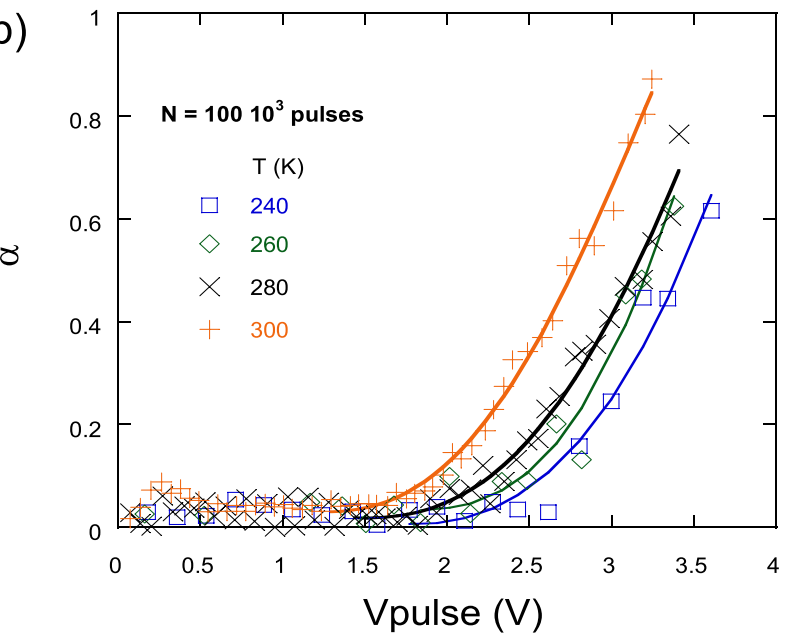

c)

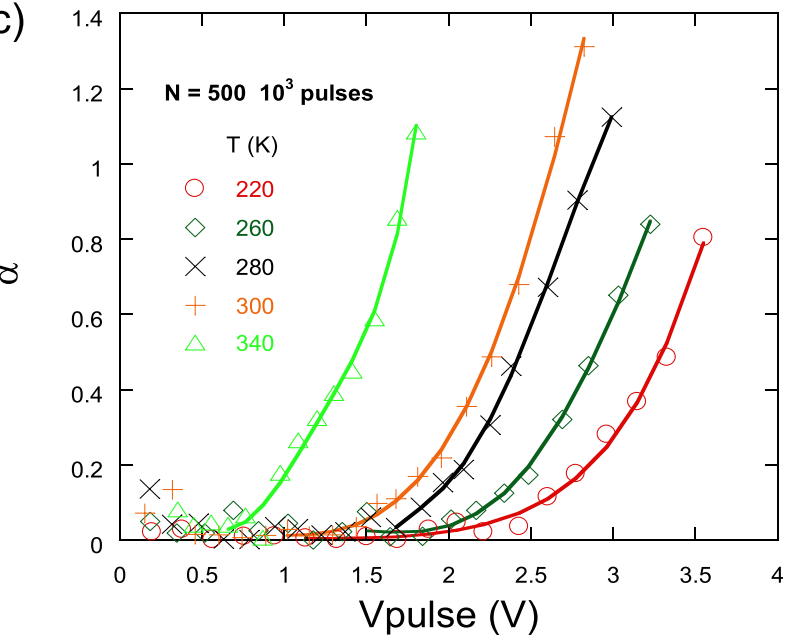

FIG. 2. Relative variation of the remanent resistance $\left(\alpha=\Delta R / R_{L}\right)$ of the $\mathrm{Au}-\mathrm{YBCO}$ interface as a function of the amplitude of the reset pulses $\left(V_{\text {pulse }}\right)$ at different temperatures. The pulse treatment corresponds to (a) $\mathrm{N}=60 \times 10^{3}$, (b) $\mathrm{N}=100 \times 10^{3}$, and (c) $\mathrm{N}=500 \times 10^{3}$ square pulses with the same characteristics described in the text. Lines are guides to the eye.

increased and decreased making that $R(a i)_{H} \gg R(a i)_{L}$. In this case, there is not a proper failure. Instead, more than a failure criteria, a value to achieve can be established, as for example $\alpha \geq \alpha_{0}$, which can be actually considered as a condition to fulfill for the reliable operation of the device. This can be the particular case of our devices, where a moderate

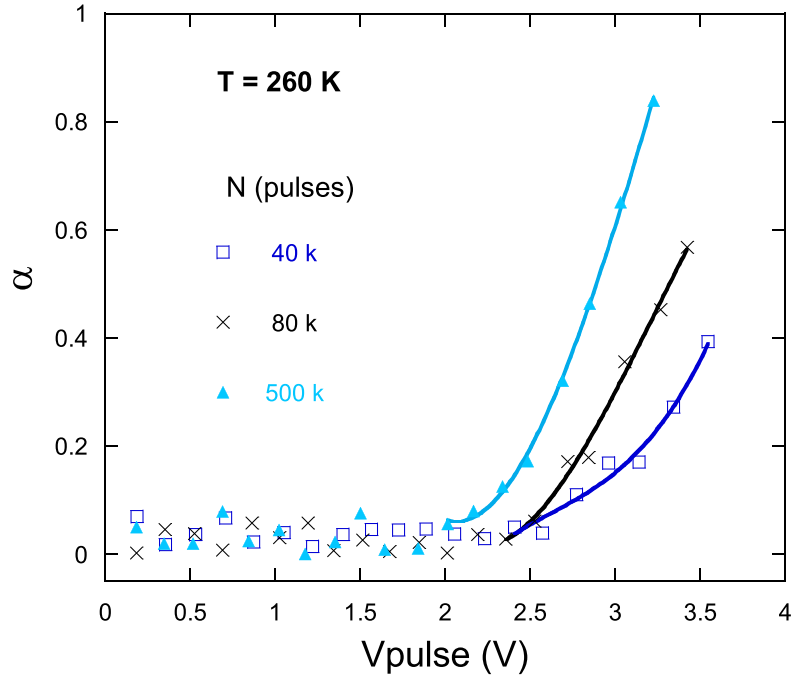

FIG. 3. $\alpha$ as a function of the amplitude of the reset pulses $\left(V_{\text {pulse }}\right)$ at $260 \mathrm{~K}$, varying the number $N$ of applied pulses. Lines are guides to the eye.

RS can be obtained $(\alpha \lesssim 1)$, with $\alpha$ increasing in the whole $V_{\text {pulse }}$ range explored.

Additional scenarios can be considered, like the case where $\alpha$ saturates, reaching a maximum, indicating that a dynamic equilibrium is established between the number of vacancies generated by the pulses and the vacancies filled with oxygen. Other possible failure scenario is the one that can be observed in unipolar devices, where a failure occurs when the low state requires a very high current to be reset. ${ }^{22}$ In this case, a proper RS failure is obtained and corresponds to $\alpha=0$ in the whole operating $V_{\text {pulse }}$ range.

As mentioned previously, in our experimental case we choose to define arbitrarily that a failure occurs when $\alpha$ reaches a predetermined value $\left(\alpha_{0}\right)$. With the results presented in Figs. 2 and 3, a typical stress-fatigue lifetime curve (or $V-N_{\alpha}$ curve), shown in Fig. 4, can be obtained as an answer to the question of which is the number of pulses $N_{\alpha_{0}}$ of amplitude $V_{\text {pulse }}$ needed to produce an arbitrarily fixed value of $\alpha=\alpha_{0}$ at a temperature $T=T_{0}(240 \mathrm{~K})$. Different values

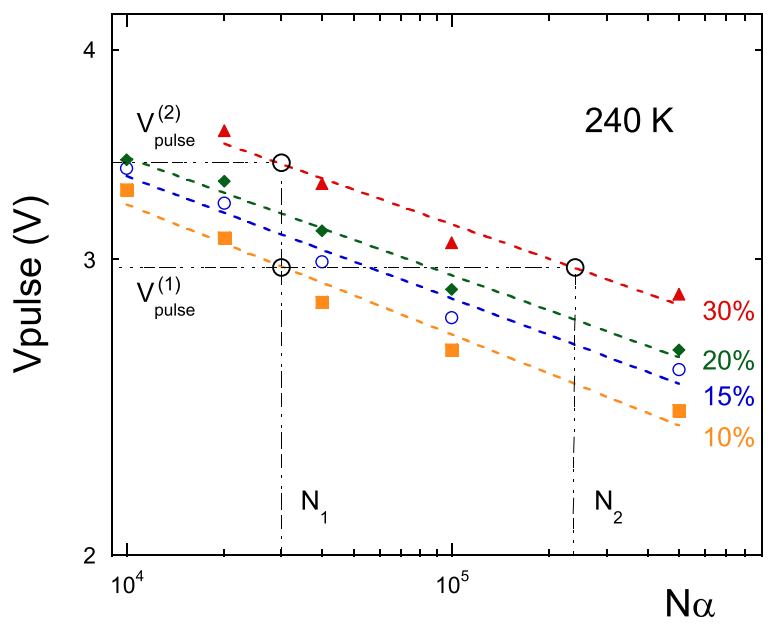

FIG. 4. Electric field stress lifetime curves (V-N curves) at $240 \mathrm{~K}$, where the failure criteria corresponds to an arbitrarily value of $\alpha=10 \%$ to $30 \%$. Dotted lines are fits corresponding to Eq. (1). Slash-dotted lines indicate two possible correction protocols to modify an obtained $\alpha_{1}=10 \%$ to a targeted $\alpha_{2}=30 \%$ (see text). 


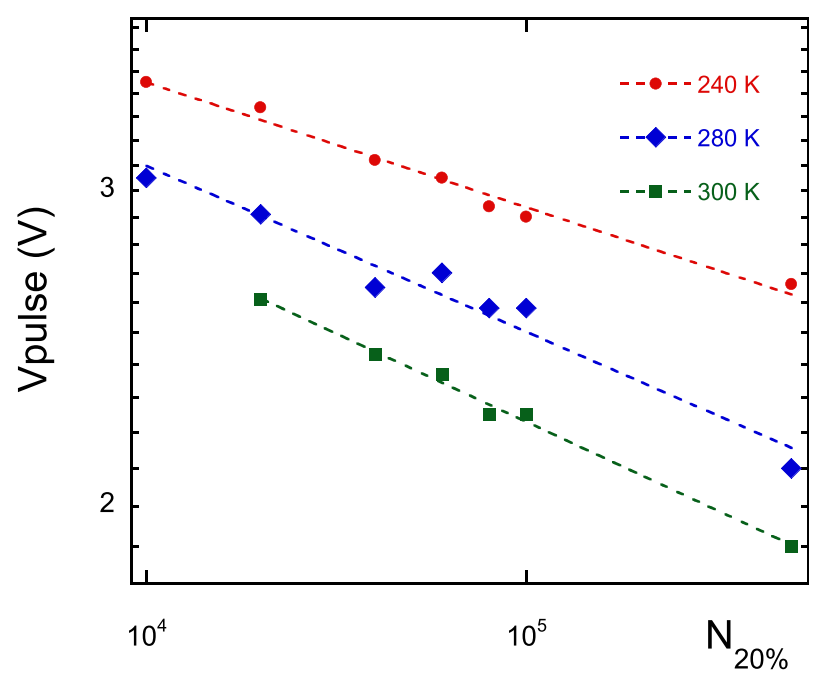

FIG. 5. V-N curves at different temperatures for a failure criteria of $20 \%$. Dotted lines are fits corresponding to Eq. (1). As for cyclic mechanical stress experiments, lowering the temperature shifts the curves to higher voltage stresses.

of $\alpha_{0}$ were considered $(10 \%, 15 \%, 20 \%$, and $30 \%)$ to check the sensitivity to its particular value. Independently of the value of $\alpha_{0}$, a power law is obtained between $V_{\text {pulse }}$ and $N_{\alpha}$, which, in fact, can be associated with the Basquin equation that describes the $S-N_{\alpha}$ curves in material fatigue experiments

$$
V_{\text {pulse }}=A N_{\text {alpha }}^{\beta},
$$

where $A$ is a constant and $\beta$ the Basquin exponent.

Independently of the failure criteria adopted, we obtained very similar curves with nearly the same exponent $-0.1 \lesssim \beta \lesssim-0.07$. Surprisingly, this particular value is typical for most of the mechanical fatigue test performed on metals. $^{12}$

If after a reset (or a set) pulsing protocol of $N_{1}$ pulses of amplitude $V_{\text {pulse }}^{(1)}$ the obtained $\alpha_{1}$ is out of the targeted range $\left(\alpha_{2} \pm \Delta \alpha\right)$, the results shown in Fig. 4 can be very useful in order to establish a first order feedback protocol to correct this issue with a single burst of pulses. By considering the sensitivity to $\alpha$ of the Basquin curves at a fixed $V_{\text {pulse }}^{(1)}$ or at a fixed number of pulses $N_{1}$, two different strategies can be envisioned: to apply a new burst of $N_{2}$ pulses at fixed $V_{\text {pulse }}^{(1)}$, or to fix the number of pulses to $N_{1}$ and modify the amplitude $V_{\text {pulse }}^{(2)}$ (see Fig. 4). In order to reach the targeted $\alpha_{2}$, it can be shown that if the voltage is kept constant, $N_{2}=(1+\epsilon)^{-\beta^{-1}} N_{1}$, while if the number of applied pulses is kept constant, the correction algorithm will be $V_{\text {pulse }}^{(2)}$ $=(1+\epsilon) V_{\text {pulse }}^{(1)}\left[\right.$ with $\left.\epsilon=\frac{\partial V_{\text {pulse }}}{\partial \alpha} \frac{\left(\alpha_{2}-\alpha_{1}\right)}{V_{\text {pulse }}^{(1)}}\right]$. If we consider the data presented in Fig. 4, in order to produce a correction to the targeted $\alpha$ of 0.2 (from 0.1 to 0.3 ), as $-\beta^{-1} \simeq 14$ we obtain that $\epsilon \simeq 0.16$, which indicates that the best strategy is to modify $V_{\text {pulse }}$ by a $16 \%$ instead of increasing $\simeq 8$ times the number of applied pulses, which increases proportionally the time needed to correct $\alpha$.
Other general result in material fatigue experiments, it is observed that, due to the temperature dependence of plastic deformation, a decrease in testing temperatures shifts the $S N_{\alpha}$ curves towards higher fatigue strengths. ${ }^{23}$ In Fig. 5, we have plotted our $V-N_{\alpha}$ curves considering a fixed $\alpha=20 \%$ where this behavior is also reproduced. Similar results were obtained for other $\alpha_{0}$ values.

A possible interpretation of these results indicates that the physics behind the electric field assisted propagation of vacancies is similar to the propagation of defects produced during a cyclic mechanical fatigue stress to a material. In fact, if a fracture can be considered as the consequence of an accumulation of interatomic bonds ruptures, our results are consistent with a framework where oxygen diffuses producing correlated defects as twins or stacking faults. As the remnant resistance of the metal-oxide interface is considered proportional to the vacancy density near the interface, ${ }^{11}$ the observed increase of $\alpha$ with the number of cycles is then a natural consequence of the oxygen vacancy production rate.

\section{CONCLUSIONS}

We have studied the sensitivity of the remnant RS change to the amplitude of cyclic voltage pulses at different temperatures and number of pulses.

We showed that if an arbitrarily fixed percentage of resistance change $\left(\alpha \geq \alpha_{0}\right)$ is associated with the failure criteria usually defined in mechanical tests, the electric field equivalent stress-fatigue lifetime curves can be obtained for a device. In this way, we provide the relation between the RS amplitude and the number of applied pulses, at a fixed amplitude and temperature. This relation can be used as the basis to build an error correction scheme. Additionally, this similarity points out that the evolution of the remnant resistance after a cyclic electric field treatment related to the process of accumulation of vacancies near the metal-oxide interface has a strong physical resemblance to the propagation of defects in materials subjected to cyclic mechanical stress tests.

\section{ACKNOWLEDGMENTS}

We would like to acknowledge the financial support by CONICET Grant PIP 112-200801-00930 and UBACyT 20020100100679 (2011-2014). We also acknowledge V. Bekeris for a critical reading, and D. Giménez, E. Pérez Wodtke, and D. Rodríguez Melgarejo for their technical assistance.

${ }^{1}$ G. W. Burr, B. N. Kurdi, J. C. Scott, C. H. Lam, K. Gopalakrishnan, and R. S. Shenoy, IBM J. Res. Dev. 52, 449 (2008).

${ }^{2}$ R. Waser and M. Aono, Nature Mater. 6, 833 (2007).

${ }^{3}$ A. Sawa, Mater. Today 11, 28 (2008).

${ }^{4}$ K. M. Kim, D. S. Jeong, and C. S. Hwang, Nanotechnology 22, 254002 (2011).

${ }^{5}$ D. S. Jeong, R. Thomas, R. S. Katiyar, J. F. Scott, H. Kohlstedt, A. Petraru, and C. S. Hwang, Rep. Prog. Phys. 75, 076502 (2012).

${ }^{6}$ C. Schindler, S. C. P. Thermadam, R. Waser, and M. N. Kozicki, IEEE Trans. Electron Devices 54, 2762 (2007).

${ }^{7}$ M. Fujimoto, H. Koyama, M. Konagai, Y. Hosoi, K. Ishihara, S. Ohnishi, and N. Awaya, Appl. Phys. Lett. 89, 223509 (2006). 
${ }^{8}$ S.-L. Li, J. Li, Y. Zhang, D.-N. Zheng, and K. Tsukagoshi, Appl. Phys. A 103, 21 (2011).

${ }^{9}$ J. J. Yang, D. B. Strukov, and D. R. Stewart, Nat. Nanotechnol. 8, 13 (2013).

${ }^{10}$ Flash Memories, edited by I. Stievano (InTech, 2011).

${ }^{11}$ M. J. Rozenberg, M. J. Sánchez, R. Weht, C. Acha, F. Gomez-Marlasca, and P. Levy, Phys. Rev. B 81, 115101 (2010).

${ }^{12}$ S. Suresh, Fatigue of Materials (Cambridge University Press, 1998).

${ }^{13}$ C. Acha and M. J. Rozenberg, J. Phys.: Condens. Matter 21, 045702 (2009).

${ }^{14}$ C. Acha, Physica B 404, 2746 (2009).

${ }^{15}$ A. Plecenik, M. Tomasek, T. Plecenik, M. Truchly, J. Noskovic, M. Zahoran, T. Rocha, M. Belogolovskii, M. Spankova, S. Chromik, and P. Kus, Appl. Surf. Sci. 256, 5684 (2010).
${ }^{16}$ C. Acha, J. Phys. D: Appl. Phys. 44, 345301 (2011).

${ }^{17}$ T. Plecenik, M. Tomasek, M. Belogolovskii, M. Truchl, M. Gregor, J. Noskovic, M. Zahoran, T. Roch, I. Boylo, M. Spankova, S. Chromik, P. Kus, and A. Plecenik, J. Appl. Phys. 111, 056106 (2012).

${ }^{18}$ A. Schulman, M. J. Rozenberg, and C. Acha, Phys. Rev. B 86, 104426 (2012).

${ }^{19}$ N. Ghenzi, M. J. Sánchez, M. J. Rozenberg, P. Stoliar, F. G. Marlasca, D. Rubi, and P. Levy, J. Appl. Phys. 111, 084512 (2012).

${ }^{20}$ W. Schutz, Eng. Fract. Mech. 54, 263 (1996).

${ }^{21}$ B. H. Moeckly, D. K. Lathrop, and R. A. Buhrman, Phys. Rev. B 47, 400 (1993).

${ }^{22}$ S. B. Lee, S. C. Chae, S. H. Chang, J. S. Lee, S. Seo, B. Kahng, and T. W. Noh, Appl. Phys. Lett. 93, 212105 (2008).

${ }^{23}$ J. Kohaut, Fatigue Fract. Eng. Mater. Struct. 23, 969 (2000). 